\title{
Incremental shuttle walk test as an indicator of decreased exercise tolerance in patients with chronic obstructive pulmonary disease
}

\author{
Jana Hodonskáa ${ }^{1, *}$, Kateřina Neumannová ${ }^{1}$, Zdeněk Svoboda ${ }^{1}$, Vratislav Sedlák $^{2}$, \\ Jaromír Zatloukal $^{3}$, Marek Plutinskýn, Vladimír Koblížek², and Lucia Bizovská ${ }^{1}$ \\ ${ }^{I}$ Faculty of Physical Culture, Palacký University Olomouc, Olomouc, Czech Republic; '2University Hospital, Hradec Králové, \\ Czech Republic; ${ }^{3}$ Faculty of Medicine and Dentistry, Palacký University Olomouc, Olomouc, Czech Republic; and ${ }^{4}$ Faculty \\ of Medicine, University Hospital of Masaryk University, Brno, Czech Republic
}

Copyright: (C) 2016 J. Hodonská et al. This is an open access article licensed under the Creative Commons Attribution License (http://creativecommons.org/licenses/by/4.0/).

\begin{abstract}
Background: Chronic obstructive pulmonary disease (COPD) is accompanied by a number of clinical symptoms, from cough and breathlessness to system disorders as a result of comorbidities. These manifestations have a significant effect on decreased exercise tolerance. Patients are limited in activities of daily living and in physical activities, especially walking, walking upstairs and uphill. Objective: The objective of this study was to assess exercise tolerance and compare the results of the Incremental shuttle walk test (ISWT) in patients with severe COPD and a group of healthy individuals. The next aim was focused on the changes in haemoglobin oxygen saturation during the test, Borg scale values and reasons for termination of the test. Methods: The research sample consisted of 17 patients (mean age $65.5 \pm 7.3$ years) with severe COPD (post-bronchodilator forced expiratory volume in one second less than $60 \%$ ). The group of healthy individuals consisted of 17 healthy individuals (mean age $62.6 \pm 2.0$ years). The participants underwent ISWT. Throughout the whole test, saturation and heart rate were monitored using a finger oximeter recorder. Results: Patients with COPD covered a significantly shorter distance in ISWT $(p<.001)$, they were subject to more significant decrease in saturation $(9 \%)$, reported subjective breathlessness more $(p<.001)$, and the test presented greater exertion for them $(14.2 \pm 3.2$ vs. $10.7 \pm 1.9)$. Conclusions: This study confirmed a decreased level of exercise tolerance in patients with severe COPD. Another conclusion of the study is that exercise assessed by means of the ISWT causes greater desaturation in COPD patients compared with healthy individuals.
\end{abstract}

Keywords: COPD, exercise tolerance, exercise testing, field walking test

\section{Introduction}

The chronic obstructive pulmonary disease (COPD) is a social issue. It is a treatable and preventable clinically heterogeneous syndrome characterized by lung damage and associated comorbidities. The disease is one of the main causes of morbidity and mortality worldwide. The estimated prevalence in the Czech Republic is $7-8 \%$ (Global initiative for chronic obstructive lung disease, 2015; Koblizek et al., 2013).

The dominating symptoms of COPD include progressive breathlessness, coughing, and in some patients expectoration. At the beginning, breathlessness shows

\footnotetext{
* Address for correspondence: Jana Hodonská, Institute of Active Lifestyle, Faculty of Physical Culture, Palacký University Olomouc, třída Míru 117, 77111 Olomouc, Czech Republic. E-mail: jana.hodonska01@upol.cz
}

after greater physical exertion, in later stages it comes during activities of daily living, or even at rest. Patients also experience extrapulmonary COPD manifestations such as the loss of fat free mass, dysfunction or atrophy of skeletal muscles, and an increased prevalence of osteoporosis. Patients with COPD are more frequently predisposed to anxiety disorders and sleep disorders. Social isolation and loss of contacts support the development of depression (Barnes \& Celli, 2009; Koblizek et al., 2013). It was confirmed that skeletal muscle dysfunction contributes to a decrease in exercise tolerance (Gosselink, Troosters, \& Decramer, 1996). From the early stages of COPD, the quadriceps femoris muscle (Seymour et al., 2010; Shrikrishna et al., 2012) and the distal muscles of the lower leg (Gagnon et al., 2013; Maddocks, Jones, et al., 2014) tend to be weakened. Persons with COPD often live a sedentary lifestyle, which supports the development of decondition. 
Physical inactivity causes worsening of the original manifestations of the disease as well as emergence of new problems. The level of exercise tolerance is determined by the results of exercise testing. In routine clinical practice it is advisable to apply a simple, yet effective and reliable exercise test.

Exercise tolerance is tested by standardized laboratory and field tests. The gold standard for the assessment of exercise tolerance and determination of respiratory and cardiac exercise limitation is spiroergometric examination. However, this examination is not performed in all patients; therefore, the assessment of exercise tolerance is more frequently performed by means of field walking tests. Field walking tests are inexpensive and do not require sophisticated equipment or special testing facilities (Holland et al., 2014). Existing studies refer especially to the Six minute walk test, during which the patient walks in a $30 \mathrm{~m}$ long corridor as fast as possible for six minutes (American Thoracic Society, 2002). Another test with less space demands is the Incremental shuttle walk test (ISWT), which requires a $10 \mathrm{~m}$ long track; the walking pace is controlled by means of audio recording (Singh, Morgan, Scott, Walters, \& Hardman, 1992). The ISWT proved to be an effective predictor of survival and mortality (Ringbaek et al., 2010). Hill et al. (2012) demonstrated a similar response in oxygen consumption and heart rate compared with laboratory testing using an ergometer.

The minimum clinically significant change in the difference between two ISWT tests to assess the effect of treatment is $47.5 \mathrm{~m}$. This value corresponds with the subjectively perceived change after a seven-week rehabilitation programme (Singh, Jones, Evans, \& Morgan, 2008).

The aim of our study was to assess exercise tolerance and compare the results of the ISWT in patients with severe COPD and a group of healthy individuals. The results of our study may contribute to further elaboration of national guidelines for the treatment of severe COPD, and suggest the ISWT as an appropriate examination test in the clinical practice of physicians and physiotherapists.

\section{Methods}

\section{Participants}

The study involved patients with COPD, who were randomly selected from the Czech multicentric research database of the COPD - Registry of the Czech Pneumological and Phthisiological Society. Patients were recruited from three registries of university hospitals in Hradec Králové, Brno and Olomouc. A precondition for participation in the study was signing an informed consent form. The study protocol and the text of the informed consent form were approved by the Ethics Committees of these university hospitals. All patients had severe COPD (post-bronchodilator forced expiratory volume in one second less than $60 \%$ ) and were in a stable condition (without exacerbation). The second research group consisted of healthy individuals from the Czech blood donor registry. The basic characteristics of the groups are shown in Table 1.

Table 1

Baseline characteristics (mean $\pm S D$ ) of participants

\begin{tabular}{lcc}
\hline & $\begin{array}{c}\text { Patients } \\
\text { with COPD }\end{array}$ & $\begin{array}{c}\text { Healthy } \\
\text { individuals }\end{array}$ \\
\hline$N$ (female/male) & $17(12 / 5)$ & $17(11 / 6)$ \\
Age $($ years $)$ & $65.5 \pm 7.3$ & $62.6 \pm 2.0$ \\
BMI $\left(\mathrm{kg} / \mathrm{m}^{2}\right)$ & $27.1 \pm 5.7$ & $28.1 \pm 3.0$ \\
$\mathrm{FEV}_{1}(\mathrm{~L} / \mathrm{s})$ & $1.3 \pm 0.4^{* *}$ & $3.0 \pm 0.8$ \\
$\mathrm{FEV}_{1}(\%$ of predicted $)$ & $46.5 \pm 14.3^{* *}$ & $99.4 \pm 16.3$ \\
$\mathrm{FVC}(\mathrm{L})$ & $2.5 \pm 0.6^{* *}$ & $3.7 \pm 0.9$ \\
$\mathrm{FVC}^{*}(\%$ of predicted $)$ & $71.7 \pm 15.0^{* *}$ & $99.8 \pm 15.8$ \\
$\mathrm{FEV}_{1} / \mathrm{FVC}$ & $51.3 \pm 11.2^{* *}$ & $80.1 \pm 4.6$ \\
\hline
\end{tabular}

Note. $\mathrm{COPD}=$ chronic obstructive pulmonary disease; $N=$ number of participants; $\mathrm{BMI}=$ body mass index; $\mathrm{FEV}_{1}=$ forced expiratory volume in one second; $\mathrm{FVC}=$ forced vital capacity; $\mathrm{FEV}_{1} / \mathrm{FVC}=$ Tiffeneau index. $\quad * * p<.001$.

Exclusion criteria were as follows: presence of severe bronchiectasis; decompensated cardiovascular disease; extrapulmonary disease, where exercise testing is contraindicated, including mobility disorders (rheumatological, neurological, psychiatric, or orthopaedic); anaemia ( $\mathrm{Hb}$ below $100 \mathrm{~g} / \mathrm{L}$ ); long-term home oxygen therapy and FeNO above 45 ppb (stable condition, at least 6 weeks without exacerbation).

\section{Measurements}

Field exercise testing

All participants underwent two rounds of the ISWT exercise test on a single day in compliance with standardized guidelines (Singh et al., 1992). The only exception was that the participants were asked to walk barefoot for the purposes of an analysis of walking during exercise testing. The $10 \mathrm{~m}$ long track was marked with cones and was located in a carpeted corridor. During the ISWT, the participants walked there and back at an increasing pace controlled by an audio recording. The test was terminated when the patient was limited by breathlessness, fatigue or pain in the lower limbs, or was terminated by the testing person if the patient was not able to keep up with the audio-controlled pace. Throughout the whole test, haemoglobin oxygen 
saturation and heart rate were monitored using a finger oximeter recorder (WristOx2, Model 3150, Nonin Medical, Plymouth, MN, USA). Each participant underwent the ISWT twice. For further processing, the better result of the two tests was used. The achieved test level and the reason for termination of the test were recorded in standardized sheets. The achieved ten-metre sections were recorded in the sheets; their sum was used for the calculation of the total distance in metres. The recording was purchased from the Pulmonary Rehabilitation Department, University Hospitals of Leicester NHS Trust, Glenfield Hospital, Leicester, United Kingdom.

Between the two tests, the participants had a rest and were allowed a drink of water. After reaching rest values of the heart rate and saturation, the second ISWT test took place. Before and after the walking exercise test, breathlessness was assessed on the Borg scale, pain in the lower limbs was reported and subjectively perceived intensity of exertion was assessed on the Borg scale.

\section{Data analysis}

Test data were written into record sheets during the course of the tests; from then on, only the outcomes of the better ISWT exercise test were used. The subsequent statistical data processing was performed using the STATISTICA programme (Version 10; StatSoft, Tulsa, OK, USA). The comparison of the results of both groups was performed using the t-test for two independent samples. Any differences at a level of statistical significance of $p<.05$ were considered significant.

\section{Results}

We observed a statistically significant difference in the results of the ISWT test between the groups (Table 2). Patients with COPD walked a significantly shorter distance by $42 \%$ (on average by $305.9 \mathrm{~m}$ ). We were also interested in the changes of the values of haemoglobin oxygen saturation. Significant differences were observed in all three measured situations (before the test, immediately after completion the test, and two minutes after completion of the test). Rest saturation was lower in patients than in healthy individuals. Our results show that patients were subject to significant (9\%) desaturation during exercise.

Before the ISWT test, none of the participants felt breathlessness or pain in the lower limbs. The average values of the Borg scale are shown in Table 3. The values include breathlessness, pain in the lower limbs and level of exertion. In the group of healthy individuals, all 17 participants terminated the test for not
Table 2

Results of ISWT test (mean $\pm S D$ )

\begin{tabular}{lcc}
\hline & $\begin{array}{c}\text { Patients } \\
\text { with COPD }\end{array}$ & $\begin{array}{c}\text { Healthy } \\
\text { individuals }\end{array}$ \\
\hline ISWD (m) & $415.3 \pm 146.3^{* *}$ & $721.2 \pm 134.9$ \\
$\mathrm{SpO}_{2}$ rest (\%) & $94.1 \pm 2.0^{* *}$ & $96.0 \pm 1.2$ \\
$\mathrm{SpO}_{2}$ end (\%) & $86.2 \pm 6.4^{* *}$ & $94.3 \pm 2.3$ \\
$\mathrm{SpO}_{2}$ recovery (\%) & $94.6 \pm 1.8^{* *}$ & $96.2 \pm 1.3$ \\
\hline
\end{tabular}

Note. $\mathrm{COPD}=$ chronic obstructive pulmonary disease; ISWD = Incremental shuttle walk distance; $\mathrm{SpO}_{2}$ rest = rest saturation before ISWT; $\mathrm{SpO}_{2}$ end = saturation immediately after ISWT; $\mathrm{SpO}_{2}$ recovery $=$ saturation measured two minutes after completion of ISWT. $* * p<.001$.

Table 3

Average resulting values of subjective assessment on the Borg scale of breathlessness and the Borg scale of exertion

\begin{tabular}{lcc}
\hline & $\begin{array}{c}\text { Patients } \\
\text { with COPD }\end{array}$ & $\begin{array}{c}\text { Healthy } \\
\text { individuals }\end{array}$ \\
\hline Dyspnea rest/end & $0 / 6^{* *}$ & $0 / 2$ \\
Leg pain rest/end & $0 / 2^{*}$ & $0 / 0$ \\
Exertion rest/end & $6 / 14^{* *}$ & $6 / 11$ \\
\hline
\end{tabular}

Note. $\mathrm{COPD}=$ chronic obstructive pulmonary disease; rest $=$ resting value; end $=$ value at the end point of the exercise . $* * p<.001, * p<.05$.

keeping up with the pace. In the group of patients with COPD, 1 patient terminated the test for breathlessness, 1 patient for pain in the calves, 13 patients for not keeping up with the pace, and the remaining 2 patients for a combination of these reasons.

\section{Discussion}

The study focused on the assessment of exercise tolerance in patients with severe COPD by means of the ISWT test. We observed a significant difference in the resulting values between the group with severe COPD and the group of healthy individuals. Patients with COPD walked a significantly shorter distance. This confirmed a decreased level of exercise tolerance in patients with pulmonary dysfunction.

Johnson-Warrington et al. (2014) performed a study, in which they compared the results of the ISWT test in patients with COPD and healthy individuals, all of whom reported subjective breathlessness of degree two according to the Modified medical research council dyspnoea scale. This degree corresponds with walking breathlessness, as a result of which, such person 
has to walk at a slower pace compared with individuals of the same age. They found a significant decrease in exercise capacity in patients with COPD. In their sample of 83 patients and 19 healthy individuals, in the ISWT test the group of patients achieved a result lower by $34 \%$. In our study, we confirmed a $42 \%$ difference between the distance achieved by patients with COPD and healthy individuals. The higher difference in our study may result from the fact that we compared healthy individuals without signs of rest breathlessness with persons with severe COPD.

During the exercise test we monitored the changes in saturation values. Patients with COPD began the test with a considerably lower level of saturation compared with the sample of healthy individuals and desaturated significantly during the test. Two minutes after completion of the test the saturation values in both groups returned to rest values. In our group of patients, three patients desaturated slightly below the value of $\mathrm{SpO}_{2}<85 \%$. This result clearly shows that the ISWT may help detect patients with COPD who may need additional oxygen treatment for exercise training (Neumannová, Zatloukal, \& Kobližek, 2014). On the other hand, use of long-term home-based oxygen therapy in these patients requires further assessment - e.g. evaluation of arterial blood gases (Koblizek et al., 2013).

None of the participants started the ISWT test with breathlessness or rest pain in the lower limbs. After completion of the walking test the group of patients were more breathless and reported more pain in the lower limbs. Exercise intolerance in patients with COPD is of a multifactorial origin. O'Donnell, Guenette, Maltais, and Webb (2012) confirmed the significance of pulmonary dysfunction. Patients with the progression of the disease were affected by dynamic hyperinflation, which prevents the lungs from increasing the breathing capacity in moments of increased demands for oxygen supply under physical exertion. Airway obstruction, decreased inspiration capacity and lung hyperinflation are associated with the change of breathing mechanics and the development of breathlessness under physical exertion. The changed breathing mechanics was confirmed also in our patients, all of them had lung hyperinflation.

The loss of physical activity in patients with COPD is associated with skeletal muscle dysfunction. A frequently discussed issue is weakening of the thigh muscles, particularly changes in the vastus lateralis muscle (Seymour et al., 2010; Shrikrishna et al., 2012). Even more significant dysfunction affects the distal muscles of the lower limb, especially the tibialis anterior muscle (Gagnon et al., 2013; Maddocks, Jones, et al., 2014). It was confirmed that a loss of intramuscular fat significantly decreases exercise tolerance. The amount of fat mass and retained skeletal muscle mass had a significant effect on the results of the ISWT and Six minute walk test (Maddocks, Shrikrishna, et al., 2014). The participants in our sample were unable to increase the speed of walking according to the level required in the ISWT test. However, the reduced ability to increase the speed of walking in patients with COPD occurred earlier (at lower speeds) than in healthy individuals. This may also be associated with the reduced fitness and dynamic hyperinflation in our sample of patients. Reduced fitness in patients with COPD had been confirmed in previous studies (Guenette, Webb, \& O’Donnell, 2012; Varga, 2015).

Based on our results we recommend the ISWT for clinical practice as an indicator of the level of exercise tolerance. Patients with severe COPD understood the test instructions well and had no difficulties performing the test. The ISWT can help monitor the effect of treatment during interim or final examinations. In research studies, the standardized ISWT test has already been used for this purpose (Egan et al., 2012; Evans, Singh, Collier, Williams, \& Morgan, 2009). Our recommendation to patients with decreased exercise tolerance is a comprehensive rehabilitation treatment focusing not only on respiratory physiotherapy techniques to influence the respiratory pattern and to maintain airway hygiene, but particularly on fitness and strength training.

The limitation of our study is the focus only on patients with severe COPD. Further studies across the whole range of COPD stages should be performed to verify the applicability of the ISWT for other stages of the disease in the Czech Republic.

\section{Conclusion}

The study assessed exercise tolerance in patients with severe COPD in comparison with a group of healthy individuals. In patients with COPD the study demonstrated decreased exercise tolerance and increased exercise desaturation. The ISWT proved to be sensitive to changes in exercise tolerance and is suitable for use in clinical practice.

\section{Acknowledgment}

The authors would like to acknowledge Petr Štěpaník, Markéta Procházková, Ondřej Unzeitig, Barbora Kobližková and David Pražák who aided in this study. This work was supported by the research project POSTUP, registration number CZ.1.07/2.3.00/30.0004, by the research grant of Novartis, and by the Palacký 
University Olomouc grants IGA_FTK_2015_006 and IGA_FTK_2015_003.

\section{Conflict of interest}

There were no conflicts of interest.

\section{References}

American Thoracic Society. (2002). ATS statement. American Journal of Respiratory and Critical Care Medicine, 166, 111-117.

Barnes, P. J., \& Celli, B. R. (2009). Systemic manifestations and comorbidities of COPD. European Respiratory Journal, 33, 1165-1185.

Egan, C., Deering, B. M., Blake, C., Fullen, B. M., McCormack, N. M., Spruit, M. A., \& Costello, R. W. (2012). Short term and long term effects of pulmonary rehabilitation on physical activity in COPD. Respiratory Medicine, 106, 1671-1679.

Evans, R. A., Singh, S. J., Collier, R., Williams, J. E., \& Morgan, M. D. L. (2009). Pulmonary rehabilitation is successful for COPD irrespective of MRC dyspnoea grade. Respiratory Medicine, 103, 1070-1075.

Gagnon, P., Maltais, F., Bouyer, L., Ribeiro, F., Coats, V., Brouillard, C., ... Saey, D. (2013). Distal leg muscle function in patients with COPD. COPD: Journal of Chronic Obstructive Pulmonary Disease, 10, 235-242.

Global initiative for chronic obstructive lung disease. (2015). Global strategy for diagnosis, management, and prevention of chronic obstructive pulmonary disease. Retrieved from http://www.goldcopd.org/guidelines-global-strategy-fordiagnosis-management.html

Gosselink, R., Troosters, T., \& Decramer, M. (1996). Peripheral muscle weakness contributes to exercise limitation in COPD. American Journal of Respiratory and Critical Care Medicine, 153, 976-980.

Guenette, J. A., Webb, K. A., \& O’Donnell, D. E. (2012). Does dynamic hyperinflation contribute to dyspnoea during exercise in patients with COPD? European Respiratory Journal, 40, 322-329.

Hill, K., Dolmage, T. E., Woon, L., Coutts, D., Goldstein, R., \& Brooks, D. (2012). A simple method to derive speed for the endurance shuttle walk test. Respiratory Medicine, 106, 1665-1670.

Holland, A. E., Spruit, M. A., Troosters, T., Puhan, M. A., Pepin, V., Saey, D., ... Singh, S. J. (2014). An official European Respiratory Society/American Thoracic Society technical standard: Field walking tests in chronic respiratory disease. European Respiratory Journal, 44, 1428-1446.

Johnson-Warrington, V., Harrison, S., Mitchell, K., Steiner, M., Morgan, M., \& Singh, S. (2014). Exercise capacity and physical activity in patients with COPD and healthy subjects classified as medical research council dyspnea scale grade 2. Journal of Cardiopulmonary Rehabilitation and Prevention, 34, 150-154.

Koblizek, V., Chlumsky, J., Zindr, V., Neumannova, K., Zatloukal, J., Zak, J., ... Pracharova, S. (2013). Chronic obstructive pulmonary disease: Official diagnosis and treatment guidelines of the Czech pneumological and phthisiological society; a novel phenotypic approach to COPD with patient-oriented care. Biomedical Papers, 157, 189-201.

Maddocks, M., Jones, M., Snell, T., Connolly, B., de WolfLinder, S., Moxham, J., \& Rafferty, G. F. (2014). Ankle dorsiflexor muscle size, composition and force with ageing and chronic obstructive pulmonary disease. Experimental Physiology, 99, 1078-1088.

Maddocks, M., Shrikrishna, D., Vitoriano, S., Natanek, S. A., Tanner, R. J., Hart, N., ... Hopkinson, N. S. (2014). Skeletal muscle adiposity is associated with physical activity, exercise capacity and fibre shift in COPD. European Respiratory Journal, 44, 1188-1198.

Neumannová, K., Zatloukal, J., \& Kobližek, V. (2014). Doporučený postup plicní rehabilitace [Recommendations for pulmonary rehabilitation]. Retrieved from http:// www.pneumologie.cz/novinka/642/doporuceny-postupplicni-rehabilitace/

O’Donnell, D. E., Guenette, J. A., Maltais, F., \& Webb, K. A. (2012). Decline of resting inspiratory capacity in COPD. Chest, 141, 753-762.

Ringbaek, T., Martinez, G., Brøndum, E., Thøgersen, J., Morgan, M., \& Lange, P. (2010). Shuttle walking test as predictor of survival in chronic obstructive pulmonary disease patients enrolled in a rehabilitation program. Journal of Cardiopulmonary Rehabilitation \& Prevention, 30, 409-414.

Seymour, J. M., Spruit, M. A., Hopkinson, N. S., Natanek, S. A., Man, W. D.-C., Jackson, A., ... Wouters, E. F. M. (2010). The prevalence of quadriceps weakness in COPD and the relationship with disease severity. European Respiratory Journal, 36, 81-88.

Shrikrishna, D., Patel, M., Tanner, R. J., Seymour, J. M., Connolly, B. A., Puthucheary, Z. A., ... Hopkinson, N. S. (2012). Quadriceps wasting and physical inactivity in patients with COPD. European Respiratory Journal, 40, 1115-1122.

Singh, S. J., Jones, P. W., Evans, R., \& Morgan, M. D. L. (2008). Minimum clinically important improvement for the incremental shuttle walking test. Thorax, 63, 775-777.

Singh, S. J., Morgan, M. D., Scott, S., Walters, D., \& Hardman, A. E. (1992). Development of a shuttle walking test of disability in patients with chronic airways obstruction. Thorax, 47, 1019-1024.

Varga, J. (2015). Mechanisms to dyspnoea and dynamic hyperinflation related exercise intolerance in COPD. Acta Physiologica Hungarica, 102, 163-175. 